\title{
Effect of annealing in two-center holographic recording
}

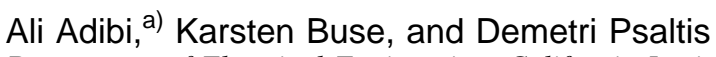 \\ Department of Electrical Engineering, California Institute of Technology, Pasadena, California 91125
}

(Received 12 March 1999; accepted for publication 28 April 1999)

\begin{abstract}
Persistent holograms are recorded with red light in lithium niobate crystals doped with manganese and iron. We find that the oxidation/reduction state of the crystal has a profound impact on the recording and readout performance. The underlying physical processes are investigated and the recording and readout responses are explained and optimized. (C) 1999 American Institute of Physics. [S0003-6951(99)05325-5]
\end{abstract}

Photorefractive materials are the best reversible storage materials known so far and several holographic storage demonstrators using iron-doped photorefractive lithium niobate $\left(\mathrm{LiNbO}_{3}: \mathrm{Fe}\right)$ were presented in the last few years. ${ }^{1-3}$ However, erasure of the holograms during readout has been one of the major problems in the practical realization of holographic read/write memories. We recently proposed a method to solve this problem by using doubly doped $\mathrm{LiNbO}_{3} .{ }^{4}$ In this letter, we explain the effects of annealing the crystal on the performance of the recently proposed twocenter recording method.

In the recent work, ${ }^{4} \mathrm{LiNbO}_{3}$ doped with manganese $(\mathrm{Mn})$ and iron $(\mathrm{Fe})$ was used. The energy band diagram of such a crystal is shown in Fig. 1. Fe and Mn ions occur in the valence states $\mathrm{Mn}^{2+/ 3+}$ and $\mathrm{Fe}^{2+/ 3+5}$, and thermal depletion plays no role in room temperature. Electrons can be excited by ultraviolet light either from $\mathrm{Mn}^{2+}$ or from $\mathrm{Fe}^{2+}$ into the conduction band while red light excites electrons only from the shallower $\mathrm{Fe}^{2+}$. The conduction-band electrons can recombine with both centers, and thus, ultraviolet illumination populates the $\mathrm{Fe}^{2+/ 3+}$ level partially while red light illumination empties the Fe sites. The filled Fe levels cause a broad-band absorption in the visible with a maximum at a $477 \mathrm{~nm}$ wavelength. ${ }^{6}$ Thus, ultraviolet light sensitizes the material while red light bleaches it. The basic idea of twocenter holographic recording is to bring with the ultraviolet light electrons from $\mathrm{Mn}$ to $\mathrm{Fe}$ via the conduction band, use these electrons to record the hologram with red light, and eventually transfer the electrons from iron back to the manganese centers by red light. This results in a hologram stored in Mn centers that persists against further red illumination.

One of the key material parameters in two-center holographic recording is the initial electron concentration in $\mathrm{Mn}$ and $\mathrm{Fe}$ traps. These concentrations can be varied by an annealing treatment. ${ }^{7}$ Since Mn traps are deeper in the band gap than Fe traps, electrons would fill the Mn traps before Fe traps when the crystal is reduced. For persistent holographic recording, it is necessary that the final hologram be stored in Mn centers. Therefore, it is essential that at the end of the annealing process all Fe traps be empty, and only a portion of the Mn traps be filled. To investigate the effect of the oxidation/reduction state of the crystal we performed experiments with four $x$-cut congruent $\mathrm{LiNbO}_{3}$ crystals doped with

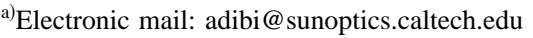

0.075 wt $\% \mathrm{Fe}_{2} \mathrm{O}_{3}$ and with $0.01 \mathrm{wt} \% \mathrm{MnO}$. The crystals were all from the same boule. The samples were strongly oxidized (LN1), oxidized (LN2), weakly oxidized (LN3), and weakly reduced (LN4) by annealing at temperatures between 700 and $1000^{\circ} \mathrm{C}$ in oxygen or argon atmosphere for different times. Sample LN1 was $2.9 \mathrm{~mm}$ thick and all others were $0.85 \mathrm{~mm}$ thick. We used a $100 \mathrm{~W}$ mercury lamp as the sensitizing light source (wavelength $365 \mathrm{~nm}$, unpolarized, intensity $14 \mathrm{~mW} \mathrm{~cm}^{-2}$ ), and a $35 \mathrm{~mW} \mathrm{HeNe}$ laser for the recording of the holograms (wavelength $633 \mathrm{~nm}$, ordinary polarization). The absorption spectra of the crystals LN1, LN3, and LN4 are shown in Fig. 2. The absorption spectrum of LN2 is very close to that of LN3, and is not shown to avoid confusion. Almost all traps in the highly oxidized crystal are empty, resulting in small absorption above $420 \mathrm{~nm}$. The absorption below this wavelength comes from the bandto-band absorption of $\mathrm{LiNbO}_{3}$, electron transfer from the valance band to $\mathrm{Fe}$ traps (hole generation), ${ }^{8}$ and possibly some absorption due to the remaining electrons in Mn traps. As we reduce the oxidized sample, more Mn traps become occupied by electrons, resulting in stronger absorption above $420 \mathrm{~nm}$ (crystals LN2 and LN3). As we continue to reduce the sample, we reach a point where all Mn traps are occupied by electrons, and start to fill Fe traps with electrons. This causes an absorption band to appear at about $477 \mathrm{~nm}$. The absorption in this band becomes stronger as we continue reducing the sample. The behavior observed in Fig. 2 implies that LN1 has hardly any electrons in either trap, LN2 and LN3 have partially filled Mn traps and empty Fe traps, and LN4 has completely filled Mn traps and partially filled Fe traps. Therefore, we expect to get poor results using either LN1 or LN4. However, LN2 and LN3 are intuitively appropriate for persistent holographic recording.

We expect that UV illumination sensitizes LN2 and LN3 for recording in red by transferring electrons from $\mathrm{Mn}$ to $\mathrm{Fe}$ traps. Experimental evidence for this effect is shown in Fig. 3 , which is the absorption spectrum of LN3 before and after UV sensitization. The occurrence of the broad absorption band at about $477 \mathrm{~nm}$ suggests electron transfer from $\mathrm{Mn}$ to Fe centers. If we illuminate the sensitized crystal with red light, all electrons in Fe traps will eventually go back to $\mathrm{Mn}$ traps, and the absorption spectrum of the crystal becomes identical to that before UV illumination. During holographic recording, UV sensitization and red bleaching are simultaneously present. To record strong holograms, we need to 


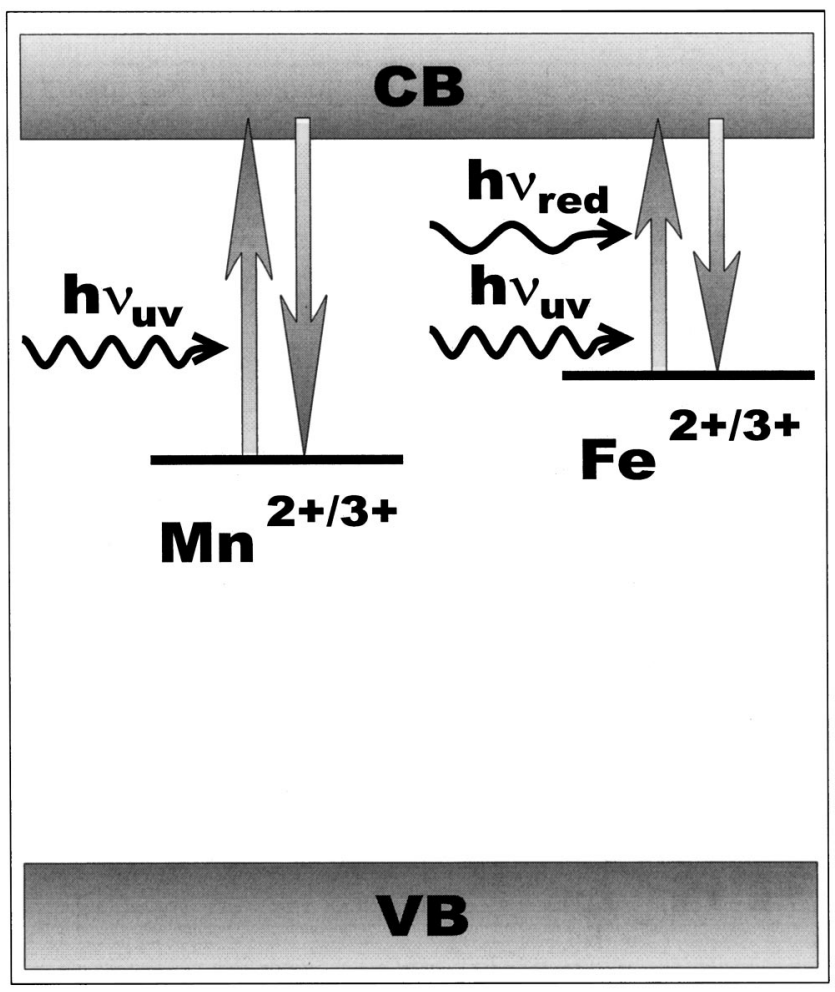

FIG. 1. Energy-band diagram for a typical $\mathrm{LiNbO}_{3}$ crystal doped with $\mathrm{Fe}$, and $\mathrm{Fe}$ and $\mathrm{Mn}$. $\mathrm{CB}$ and VB stand for conduction band and valance band, respectively.

have an optimum balance between these two effects. Too strong UV illumination causes rapid erasure of the hologram, while too strong red illumination results in too few electrons at the Fe traps, and hence, a weak hologram. The optimum balance between sensitization and bleaching is typically done by choosing a correct red-to-UV intensity ratio. Due to large UV absorption of the crystal, the optimum intensity ratio is broad. Besides the intensity ratio, the oxidation/reduction state of the crystal plays an important role. It is necessary to choose the right ratio between the concentration of the filled and empty deep $(\mathrm{Mn})$ traps to record strong persistent holograms.

To check the intuitive arguments mentioned above, we recorded holograms in the four crystals. The crystals were

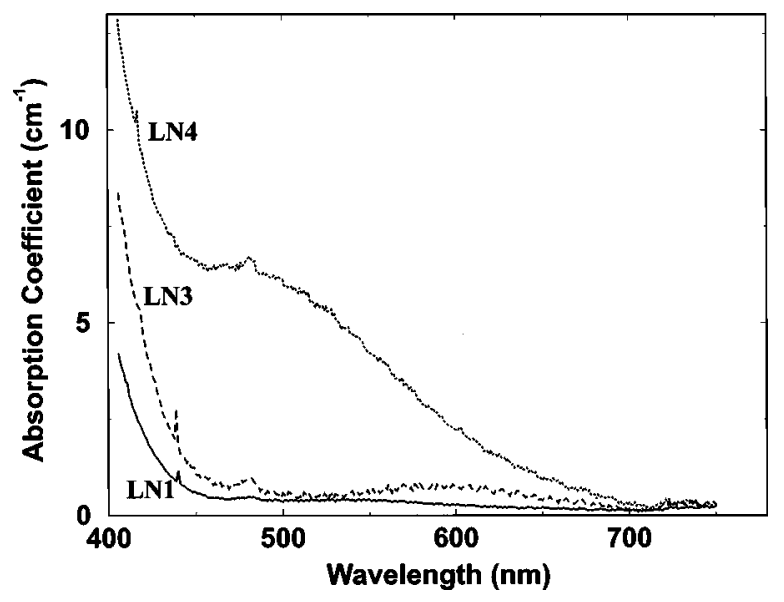

FIG. 2. Absorption spectra for the differently annealed crystals LN1, LN3, and LN4 before sensitization. The effect of Fresnel reflections has been subtracted from the spectra. LN1, LN2, LN3, and LN4.
Downloaded from the spectra.
Dow Aug 2009 to 128.178.48.60. Redistribution subject to AIP license or copyrigh

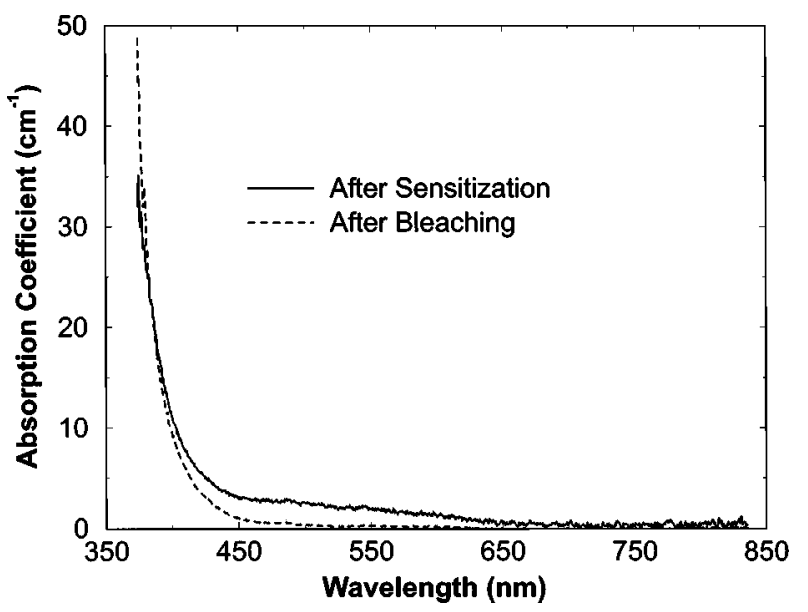

FIG. 3. Absorption spectra for the crystal LN3 before and after UV illumination. The UV intensity was $4 \mathrm{~W} / \mathrm{cm}^{2}$.

homogeneously preexposed to ultraviolet light for at least $1 \mathrm{~h}$ before each experiment. Plane-wave gratings were then recorded and reconstructed. The ultraviolet light illuminated the samples homogeneously; the HeNe laser light was split into two plane waves, which interferred at the crystal $\left(1 / e^{2}\right.$ beam diameter $2.0 \mathrm{~mm}$, transmission geometry, period length of the grating $0.9 \mu \mathrm{m}$, intensity of each beam 250 $\mathrm{mW} \mathrm{cm}{ }^{-2}$ ). The grating vector was aligned parallel to the $c$ axis of the sample. During recording, one of the HeNe beams was blocked from time to time and the second beam was diffracted from the written grating to obtain the diffraction efficiency $\eta$ as the ratio between diffracted and total incident light powers. After recording, UV and one of the red beams are blocked and readout is performed with the other red beam.

Recording and readout curves for the four crystals are shown in Fig. 4. Strong holograms cannot be recorded in LN1 as shown in Fig. 4. This is because LN1 is a highly oxidized sample. Due to the strong oxidation of LN1, only a few electrons are available in Mn traps while Fe traps are completely empty in this sample. The small number of electrons available in either trap explains the very small diffraction efficiency obtained for LN1. Persistent holograms can be recorded in both LN2 and LN3 with good diffraction efficiencies as shown in Fig. 4. We think that this is due to

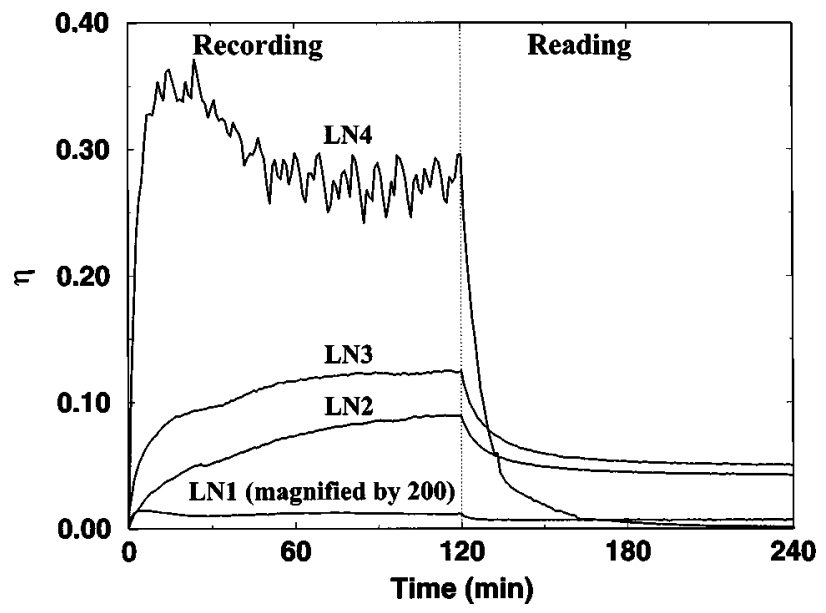

FIG. 4. Recording and erasure curves for the differently annealed crystals

AIP'license or copyright; see http://apl.aip.org/apl/copyright.jsp 
the appropriate oxidation/reduction state of these two samples. Compared to LN1, both LN2 and LN3 are reduced with stronger reduction for LN3. Therefore, LN2 and LN3 have more trapped electrons than LN1. Before sensitization, these extra electrons are in the Mn traps as discussed previously. The reduction of LN2 and LN3 is not strong enough to fill all the Mn traps, and as a result, there are no available electrons to fill the Fe traps. Therefore, it is not possible to record any holograms with only red light in LN2 and LN3 without UV sensitization. However, electrons can be transferred to the Fe traps by UV light. This is due to both having more electrons in Mn traps and fewer empty Mn centers to trap electrons from the conduction band. The latter results in a larger probability of electron trapping at Fe centers. Therefore, we can record faster and get larger diffraction efficiencies. During readout by red light, electrons are transferred from Fe traps to $\mathrm{Mn}$ traps resulting in a partial erasure of the hologram. When all electrons are transferred to Mn centers, the remaining hologram persists against further readout. Therefore, reducing the crystal results in an increase in both sensitivity and the $\mathrm{M} / \# .{ }^{9}$ This explains the recording and readout curves for both LN2 and LN3. Since LN3 is more reduced than $\mathrm{LN} 2$, it has faster recording and stronger final diffraction efficiency. The recording curve for LN4 is also shown in Fig. 4. It is much faster than the other samples and larger saturation diffraction efficiencies are obtained. However, the recorded hologram is erased during readout by red light only. This is because LN4 is a strongly reduced sample. When the crystal is reduced too much, we reach a point where we do not have enough empty Mn traps to store a strong space-charge pattern, although we have a lot of electrons. Therefore, we can record strong holograms with good speed, but we lose a major part of it during readout. We conclude that the final persistent diffraction efficiency is limited by the availability of empty Mn traps before sensitization. When we reduce the crystal so strongly that all the Mn traps are filled, then we lose the persistence property completely. This is the case for LN4. In this case, we have very good sensitivity (fast recording) because LN4 is reduced so much that the Fe traps are partially filled as well. We can also record strong holograms due to initial electron population in Fe traps and very effective UV sensitization due to filled Mn traps. However, electrons are transferred during readout from $\mathrm{Fe}$ centers to $\mathrm{Mn}$ centers until all $\mathrm{Mn}$ centers are occupied. The remaining hologram resides in Fe centers and is totally erased by further readout. The final diffraction efficiency after extensive readout is zero. This explains the recording and readout curve for LN4.

As the experimental results show, there is an optimum oxidation/reduction state for a doubly doped $\mathrm{LiNbO}_{3}$ crystal that results in the desired performance. This optimum depends on the doping levels of the shallower $(\mathrm{Fe})$ and deeper (Mn) traps and the intensities of the sensitizing (UV) and recording (red) beams. Figure 5 shows the theoretical calculation of the persistent $\mathrm{M} / \#$ (after sufficient readout) as a function of the portion of Mn traps that are initially filled. All Fe traps are initially empty. The calculation is based on

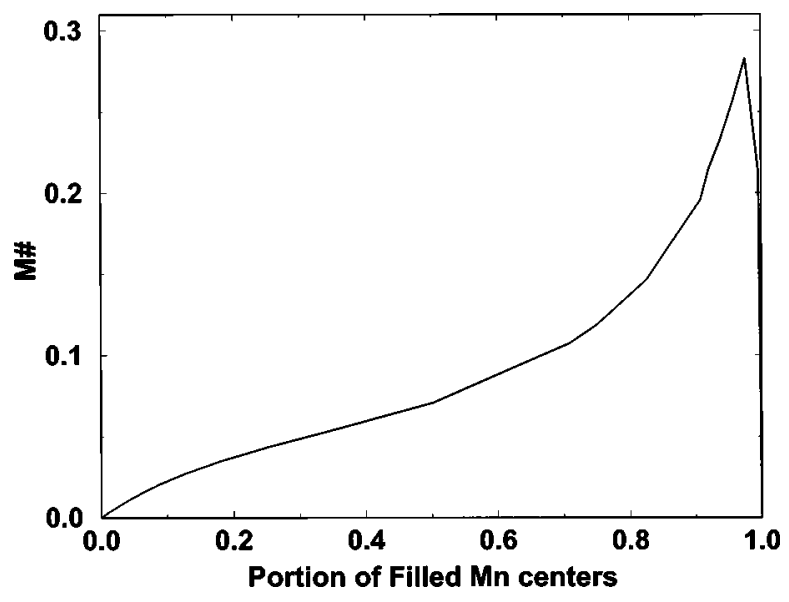

FIG. 5. Variation of the persistent $\mathrm{M} / \#$ with the portion of filled Mn traps for a $\mathrm{LiNbO}_{3}$ crystal doped with $0.075 \mathrm{wt} \% \mathrm{Fe}_{2} \mathrm{O}_{3}$ and $0.01 \mathrm{wt} \% \mathrm{MnO}$. All Fe traps are assumed to be initially empty.

the solution of the Kukhtarev equations for the two-center method. The doping levels used in this calculation are the same as those of the crystals used in the experiments. Figure 5 shows that for the crystal used in these experiments, the optimum oxidation/reduction state that results in the best $\mathrm{M} / \#$ is when about $95 \%$ of the Mn traps are filled with electrons. This is close to the oxidation/reduction state of LN3.

In conclusion, we presented a method for recording persistent holograms in doubly doped $\mathrm{LiNbO}_{3}$ crystals. We showed the effect of the oxidation/reduction state of the crystal on recording and readout responses. The experimental results confirm our explanation of the two-center holographic recording method. They also prove that there is an optimum oxidation/reduction state resulting in the best persistent $\mathrm{M} / \#$. It turns out that recording in doubly doped crystals with the simultaneous presence of long-wavelength recording and short-wavelength sensitizing light is a promising approach for all-optical nonvolatile holographic data storage, that the underlying processes are correctly understood, and that tailoring of the material and the experimental conditions enables further improvements of the performance.

This work was supported by AF/Rome Lab Award No. F0060297C0049, and by a JPL work order funded by DARPA/ITO. One of the authors (K.B.) thanks the Deutsche Forschungsgemeinschaft for a postdoctoral fellowship.

${ }^{1}$ F. H. Mok, Opt. Lett. 18, 915 (1993).

${ }^{2}$ I. McMichael, W. Christian, D. Pletcher, T. Y. Chang, and J. H. Hong, Appl. Opt. 35, 2375 (1996).

${ }^{3}$ J. Ashley, M.-P. Bernal, M. Blaum, G. W. Burr, H. Coufal, R. K. Grygier, H. Günter, J. A. Hoffnagle, C. M. Jefferson, R. M. MacFarlane, B. Marcus, R. M. Shelby, G. T. Sincerbox, and G. Wittmann, Laser Focus World 32, 81 (1996).

${ }^{4}$ K. Buse, A. Adibi, and D. Psaltis, Nature (London) 393, 665 (1998).

${ }^{5}$ O. Thiemann and O. F. Schirmer, Proc. SPIE 1018, 18 (1988).

${ }^{6}$ H. Kurz, E. Krätzig, W. Keune, H. Engelmann, U. Gonser, B. Dischler, and A. Räuber, Appl. Phys. 12, 355 (1977).

${ }^{7}$ W. Phillips, J. J. Amodei, and D. L. Staebler, RCA Rev. 33, 94 (1972).

${ }^{8}$ R. Orlowski and E. Krätzig, Solid State Commun. 27, 1351 (1978).

${ }^{9}$ F. H. Mok, G. W. Burr, and D. Psaltis, Opt. Lett. 21, 896 (1993). 